\title{
Erratum to: Averaging and Linear Programming in Some Singularly Perturbed Problems of Optimal Control
}

\author{
Vladimir Gaitsgory • Sergey Rossomakhine
}

Published online: 12 August 2014

(C) Springer Science+Business Media New York 2014

\section{Erratum to: Appl Math Optim \\ DOI 10.1007/s00245-014-9257-1}

The proof of Theorem 3.14 is based on several lemmas, one of which is Lemma 5.3. The argument used in the proof of the latter is based on the fact that, for any $(\bar{u}, \bar{y}) \in \operatorname{cl} \theta_{t}^{*}$, where

$$
\theta_{t}^{*} \stackrel{\text { def }}{=}\left\{(u, y):(u, y)=\left(u_{t}^{*}(\tau), y_{t}^{*}(\tau)\right) \text { for some } \tau \in[0, \infty)\right\}
$$

$\left(\left(u_{t}^{*}(\tau), y_{t}^{*}(\tau)\right)\right.$ being the admissible pair of the associated system introduced in Assumption 3.11(iii)),

$$
\mu^{*}(t)\left(B_{r}(\bar{u}, \bar{y})\right)>0 \quad \forall r>0
$$

where $\mu^{*}(t)$ is the occupational measure generated by $\left(u_{t}^{*}(\tau), y_{t}^{*}(\tau)\right)$ and $B_{r}(\bar{u}, \bar{y}) \stackrel{\text { def }}{=}$ $\{(u, y):\|u-\bar{u}\|+\|y-\bar{y}\|<r\}(\operatorname{see}(5.55))$.

The online version of the original article can be found under doi:10.1007/s00245-014-9257-1.

\footnotetext{
V. Gaitsgory $(\varangle)$

Department of Mathematics, Macquarie University, Sydney, NSW 2109, Australia

e-mail: vladimir.gaitsgory@mq.edu.au

S. Rossomakhine

Flinders Mathematical Sciences Laboratory, School of Computer Science,

Engineering and Mathematics, Flinders University, GPO Box 2100, Adelaide,

SA 5001, Australia

e-mail: serguei.rossomakhine@flinders.edu.au
} 
The proof of (1.1) offered in the paper is valid, however, only under the assumption that the pair $\left(u_{t}^{*}(\tau), y_{t}^{*}(\tau)\right)$ is periodic. That is,

$$
\left(u_{t}^{*}(\tau), y_{t}^{*}(\tau)\right)=\left(u_{t}^{*}\left(\tau+T_{t}\right), y_{t}^{*}\left(\tau+T_{t}\right)\right) \forall \tau \geq 0
$$

for some $T_{t}>0$.

This observation readily leads to the conclusion that, for the statement of Theorem 3.14 to be valid, one needs to replace the assumption about the validity of (3.67) by the periodicity assumption (1.2), in which $T_{t}$ should be uniformly bounded for almost all $t \geq 0$. Note that the statement of the theorem can be also proved to be valid in case one just assumes (in additions to the assumptions made in the paper) that (1.1) is valid for any $(\bar{u}, \bar{y}) \in \theta_{t}^{*}$ and for almost all $t \geq 0$. 